\title{
Influence de l'interface fibre/matrice sur le comportement jusqu'à rupture d'un polypropylène renforcé de fibres de verre courtes
}

\author{
Éric SÉgard ${ }^{a}$, Salim Benmedakhene, Abdel Laksimi et Dawei LaÏ
}

Université de Technologie de Compiègne, Centre de recherche de Royallieu, Laboratoire Roberval UMR 6066-CNRS, Polymères et Composites, BP 20529, 60205 Compiègne Cedex, France

Reçu le 18 juillet 2003, accepté le 2 décembre 2004

\begin{abstract}
Résumé - Nous présentons dans cet article une étude expérimentale de l'influence de la qualité de l'interface fibre/matrice sur le comportement en traction et en fluage jusqu'à rupture d'un polypropylène renforcé de $40 \%$ en masse de fibres de verre courtes (soit 18,85\% de fraction volumique de fibres). Les essais de traction avec des taux de fibre de 20 et $30 \%$ en masse montrent l'existence d'un plateau pour le matériau avec fibres non-traitées (absence d'ensimage) qui disparaît pour le taux de $40 \%$ en masse. Également, ces essais de traction montrent, pour chaque type d'interface, l'existence de trois zones de comportement pour chaque matériau. La mesure de l'émission acoustique permet de quantifier les différents mécanismes d'endommagement et montre qu'il y a plus d'événements acoustiques pour le matériau avec fibres traitées. Des essais de fluage jusqu'à rupture sont entrepris avec la mesure de l'émission acoustique pour un taux de fibre de $40 \%$ et montrent également plus d'événements acoustiques pour le matériau avec fibres traitées. Finalement on observe, à partir des résultats de l'émission acoustique, l'influence de la nature de la sollicitation sur les mécanismes d'endommagement et on constate qu'il y a plus d'événement lors des essais de fluage jusqu'à rupture.
\end{abstract}

Mots clés : Polypropylène / traction / fluage / interface / émission acoustique / endommagement

Abstract - Influence of the fibre/matrix interface on the behaviour until fracture of polypropylene reinforced with short glass fibres. This paper concerns an experimental study of the influence of the fibre/matrix interface on the behaviour in tensile and creep tests until fracture of polypropylene reinforced by $40 \%$ short glass fibres in mass. The tensile tests with fibre rates of $20 \%$ and $30 \%$ in mass show a plateau for the material with untreated fibres which disappears for the $40 \%$ rate (or $18.85 \%$ of fibres rate in volume). These tensile tests show, for each type of interface, three zones of behaviour for each material. The acoustic emission activities allows to quantify the various damage mechanisms and shows there are more acoustic events for material with treated fibres. Creep tests until fracture were carried out with the acoustic emission technique for $40 \%$ fibres rate these test show more acoustic events for material with treated fibres. Finally, we observe through the results of acoustic emission, the influence of the type of the mechanical test on the damage mechanisms and it can be noticed there are more events during the creep tests until fracture.

Key words: Polypropylene / tensile / creep / interface / acoustic emission / damage

\section{Introduction}

Ce travail porte sur l'influence de la qualité de l'interface fibre/matrice sur le comportement en fluagerecouvrance d'un polypropylène renforcé de $40 \%$ en masse de fibres de verre courtes au-dessus de la température de transition vitreuse $T_{\mathrm{g}}$ qui est $0{ }^{\circ} \mathrm{C}$. Le fait

\footnotetext{
a Auteur correspondant : eric.segard@utc.fr
}

d'être au-dessus de $T_{\mathrm{g}}$ implique l'apparition du comportement viscoélastique du polypropylène. L'intérêt de l'utilisation de ce matériau réside dans son faible coût de transformation pour des applications grands public (exemple de l'automobile) et surtout dans le fait qu'il soit facilement recyclable Peu de travaux ont été réalisés jusqu'à présent sur le sujet. Bounouas [1] montre, dans sa thèse, l'influence de la qualité de l'interface fibre/matrice sur le comportement en traction, en torsion et au choc pour 
Tableau 1. Paramètres moyens de traction du matériau avec fibres traitées pour les taux de fibre en masse de $20 \%$, $30 \%$ et $40 \%$.

\begin{tabular}{ccccccc}
\hline $\begin{array}{c}\text { Taux } \\
\text { de fibres }\end{array}$ & $\begin{array}{c}\text { Module } \\
\text { de Young }(\mathrm{MPa})\end{array}$ & Écart-type & $\begin{array}{c}\text { Contrainte maxi } \\
(\mathrm{MPa})\end{array}$ & Écart-type & $\begin{array}{c}\text { Déformation } \\
\text { maxi à rupture }\end{array}$ & Écart-type \\
\hline $20 \%$ & 5150 & 300 & 62,87 & 6,73 & 0,0156 & 0,0036 \\
$30 \%$ & 6810 & 410 & 70,46 & 3,67 & 0,0153 & 0,0025 \\
$40 \%$ & 9410 & 350 & 73,25 & 5,03 & 0,0123 & 0,003 \\
\hline
\end{tabular}

ce type de matériau, et notamment une meilleure tenue mécanique pour le matériau avec fibres traitées (fibres ensimées). Suite aux travaux de L. Bounouas, une étude a été menée par Ségard [2-4] en fluage-recouvrance afin d'identifier le comportement de chaque matériau, en utilisant le modèle de Schapery et de Zappas-Crisman pour décrire respectivement la viscoélasticité non-linéaire ainsi que la viscoplasticité. Il a été démontré que le niveau de déformation viscoélastique est plus élevé que le niveau de déformation viscoplastique, pour le même niveau de contrainte, pour les deux matériaux (et pour le matériau non-traité un niveau de déformation viscoélastique et viscoplastique plus élevé). On se propose dans cet article, de montrer dans un premier temps, l'influence du taux de fibre sur le comportement en traction, puis l'influence de la qualité de l'interface sur les différents mécanismes d'endommagement en s'appuyant sur la mesure de l'émission acoustique. Des essais de fluage jusqu'à rupture ont été ensuite réalisés dans le but d'observer, tout comme pour les essais de traction, l'influence de la qualité de l'interface sur les mécanismes d'endommagement. L'influence du type de sollicitation sur les différents mécanismes d'endommagement est également observée.

\section{2 Études expérimentales}

\subsection{Essais de traction}

Les essais de traction monotone ont pour but de montrer, dans un premier temps, l'influence du taux de fibres sur le comportement en traction pour le matériau avec fibres traitées ainsi que non-traitées, afin de faire le choix du taux de fibres optimal pour la suite de l'étude parmi les 3 taux de fibres à notre disposition $20 \%, 30 \%$ et $40 \%$. Dans un deuxième temps, il sera mis en évidence l'influence de la qualité de l'interface fibre/matrice sur le comportement en traction monotone ainsi qu'en fluage des matériaux étudiés ainsi que sur les mécanismes d'endommagement.

\subsubsection{Conditions d'essais}

Le matériau étudié est un polypropylène chargé à $20 \%, 30 \%$ ou $40 \%$ en masse de fibres de verre courtes avec une bonne et une mauvaise qualité d'interface fibre/matrice. Les éprouvettes sont de type haltère. Les essais de traction monotone sont réalisés à température ambiante et constante $\left(19{ }^{\circ} \mathrm{C}\right)$, sur une machine de

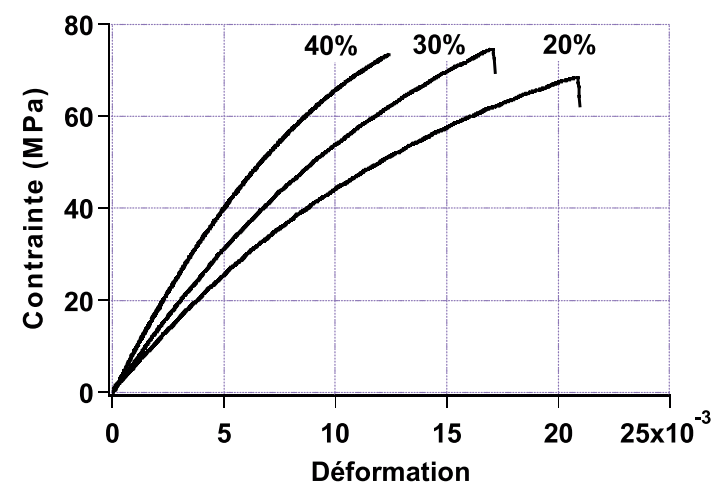

Fig. 1. Courbes de traction du matériau avec fibres traitées pour les taux de fibres en masse de $20 \%, 30 \%$ et $40 \%$.

type Instron 4411 avec une vitesse de déformation de $1,15 \times 10^{-04} \mathrm{~s}^{-1}$. Cinq essais de traction pour chaque type de matériau sont effectués. On réalise les essais de traction uniquement pour le taux de $40 \%$, les autres courbes provenant des travaux de Bounouas [1]. Le suivi de l'endommagement est réalisé à l'aide de la mesure de l'émission acoustique. Pour cela, on place un capteur piézoélectrique à la surface du matériau afin de détecter les ondes acoustiques engendrées par l'endommagement du matériau. Les éprouvettes sont équipées de jauges d'extensiométrie collées à la surface de chaque éprouvette.

\subsubsection{Résultats et discussion}

\subsubsection{Influence du taux de fibre sur le comportement en traction}

Les courbes de traction monotone jusqu'à rupture pour un taux de fibre de $20 \%, 30 \%$ et $40 \%$ pour le matériau avec fibres traitées sont présentées sur la figure 1.

Les paramètres mesurés à partir de ces essais sont résumés dans le tableau 1.

Les courbes de traction monotone jusqu'à rupture pour un taux de fibre de $20 \%, 30 \%$ et $40 \%$ pour le matériau avec fibres non traitées sont montrées dans la figure 2 .

Les paramètres de traction mesurés sont résumés dans le tableau 2.

Les courbes de traction et les tableaux des propriétés mécaniques montrent, pour les deux matériaux que le module de Young augmente avec le taux de fibres.

Pour le matériau avec fibres de verre non-traitées, on constate que la contrainte maximale et la déformation 
Tableau 2. Paramètres moyens de traction du matériau avec fibres non-traitées pour des taux de fibre en masse de $20 \%$, $30 \%$ et $40 \%$.

\begin{tabular}{ccccccc}
\hline $\begin{array}{c}\text { Taux } \\
\text { de fibres }\end{array}$ & $\begin{array}{c}\text { Module } \\
\text { de Young (MPa) }\end{array}$ & Écart-type & $\begin{array}{c}\text { Contrainte maxi } \\
(\mathrm{MPa})\end{array}$ & Écart-type & $\begin{array}{c}\text { Déformation } \\
\text { maxi à rupture }\end{array}$ & Écart-type \\
\hline $20 \%$ & 5430 & 490 & 33,09 & 1,9 & 0,023 & 0,0025 \\
$30 \%$ & 6880 & 640 & 32,7 & 1,07 & 0,012 & 0,006 \\
$40 \%$ & 9100 & 670 & 34,35 & 1,4 & 0,0062 & 0,005 \\
\hline
\end{tabular}

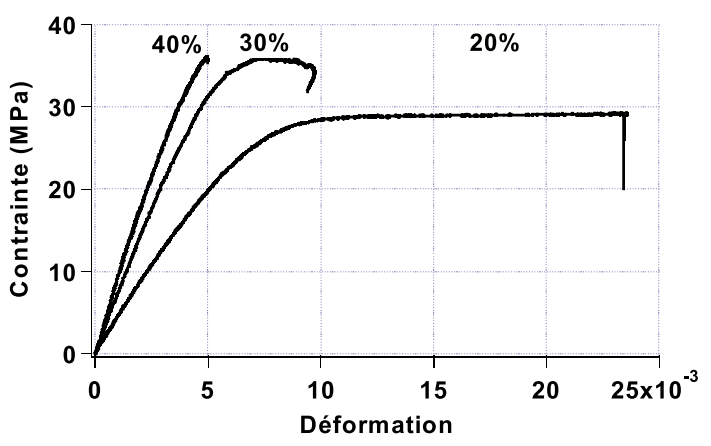

Fig. 2. Courbes de traction du matériau avec fibres nontraitées pour les taux de fibres en masse de $20 \%, 30 \%$ et $40 \%$.

à rupture évoluent très faiblement lorsqu'on dépasse un seuil critique de taux de fibre. En effet, lorsqu'on passe du taux de $30 \%$ à $40 \%$ de taux de fibres, on observe une très faible évolution de la contrainte maximale et de la déformation à rupture, ceci comparé au passage du taux de fibres de $20 \%$ à $30 \%$.

De plus, sur la courbe de traction du matériau avec $20 \%$ de fibres non-traitées, on observe un plateau caractérisé par une contrainte constante. Ce plateau diminue lorsqu'on passe à un taux de fibres de $30 \%$ pour quasiment disparaître lorsqu'on atteint le taux de $40 \%$. Ce plateau a été observé lors d'essais de traction monotone sur du polypropylène vierge [1] et provient des phénomènes liés à la déformation de la matrice. Ainsi, plus le taux de fibres est élevé, meilleur est le transfert de charge de la matrice vers les fibres. En effet, le transfert de charge matrice/fibres se faisant par la contrainte de cisaillement à l'interface, plus il y a de fibres, meilleur est ce transfert de charge. Ce transfert de charge se fait donc intégralement pour le taux de fibre de $40 \%$. Par conséquent, la contrainte maximale ainsi que la déformation à rupture n'évoluent plus pour un taux de fibres supérieur à $40 \%$ en masse.

Pour le matériau avec fibres de verre traitées, on constate qu'il n'y a pas de plateau comme pour le matériau avec fibres non-traitées et que la contrainte maximale évolue très faiblement lorsqu'on passe de $30 \%$ à $40 \%$ de taux de fibres comparé au passage de $20 \%$ à $30 \%$. La même remarque peut être faite pour le matériau avec fibres non-traitées. La contrainte maximale ainsi que la déformation à rupture n'évolueront quasiment plus pour un taux de fibres supérieur à $40 \%$ en masse.

Compte tenu de toutes ces observations faites sur les essais de traction sur le matériau avec fibres traitées et non-traitées, on conclut que le taux de fibres de

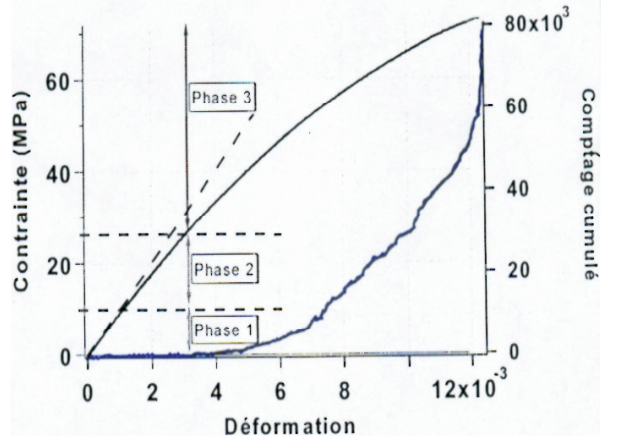

Fig. 3. Différentes phases caractérisant le comportement pour un matériau avec $40 \%$ en masse de fibres traitées.

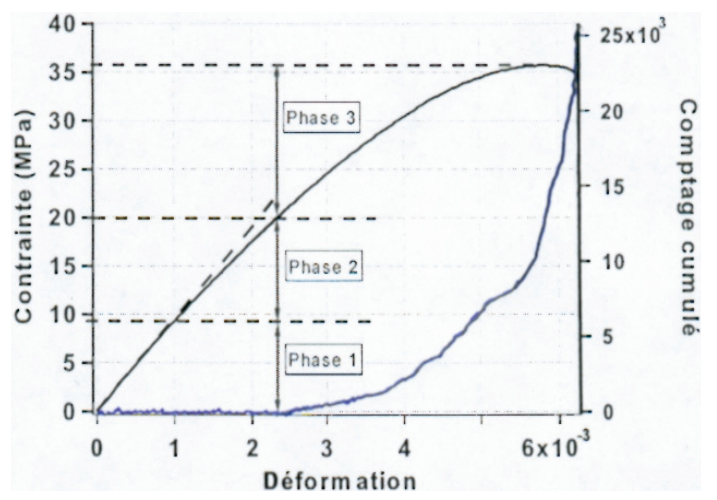

Fig. 4. Différentes phases caractérisant le comportement pour un matériau avec $40 \%$ en masse de fibres non-traitées.

verre courtes permettant d'avoir les meilleures propriétés mécaniques parmi les trois proposés est le taux de $40 \%$ en masse pour le matériau avec fibres traitées et non-traitées, soit 18,85\% de fraction volumique de fibres. C'est ce taux de fibres qui sera utilisé pour tout le reste de l'étude.

\subsubsection{Influence de la qualité de l'interface fibre/matrice sur le comportement en traction pour un taux de $40 \%$ en masse de fibre}

Les résultats des essais de traction pour chaque matériau sont présentés sur la figure 3 et la figure 4 .

Pour chaque matériau, le comportement présente trois phases distinctes qui sont : linéaire sans endommagement, non-linéaire sans endommagement puis non-linéaire avec endommagement.

Si on compare les propriétés mécaniques des deux matériaux vues précédemment sur les tableaux 1 et 2 , on peut voir que le module de Young pour le matériau avec fibres traitées et non-traitées est respectivement de 
Tableau 3. Nombre d'événements et taux de participation par plage d'amplitude pour les matériaux avec un taux de $40 \%$ en masse de fibres traitées et non-traitées soumis à un essais de traction jusqu'à rupture.

\begin{tabular}{lcccc} 
& \multicolumn{2}{c}{ Matériau avec fibres traitées } & \multicolumn{2}{c}{ Matériau avec fibres non-traitées } \\
\cline { 2 - 5 } & $\begin{array}{c}\text { Nombre } \\
\text { d'événements }\end{array}$ & $\begin{array}{c}\text { Taux } \\
\text { de participation }\end{array}$ & $\begin{array}{c}\text { Nombre } \\
\text { d'événements }\end{array}$ & $\begin{array}{c}\text { Taux } \\
\text { de participation }\end{array}$ \\
\hline $33-45 \mathrm{~dB}$ & 51041 & $64,34 \%$ & 17543 & $68,73 \%$ \\
$45-58 \mathrm{~dB}$ & 26254 & $33,1 \%$ & 7367 & $28,86 \%$ \\
$58-68 \mathrm{~dB}$ & 1730 & $2,18 \%$ & 461 & $1,81 \%$ \\
$68-86 \mathrm{~dB}$ & 197 & $0,25 \%$ & 96 & $0,38 \%$ \\
$86-100 \mathrm{~dB}$ & 102 & $0,13 \%$ & 57 & $0,22 \%$ \\
Total & 79324 & $100 \%$ & 25524 & $100 \%$ \\
\hline
\end{tabular}

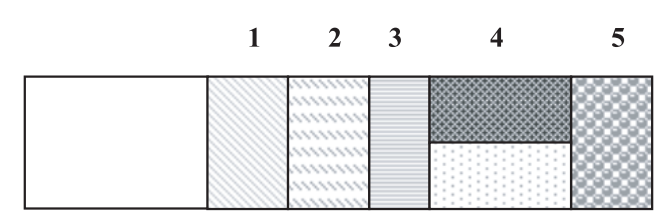

$1 \quad 33-45 \mathrm{~dB}$ : Microfissuration de la matrice

$246-58 \mathrm{~dB}$ Coalescence des microfissures

$359-68 \mathrm{~dB}$ Rupture d'interface

4 69-86 dB Déchaussement des fibres, frottement fibre-matrice

5 87-100 dB: Rupture des fibres

Fig. 5. Association des plages d'amplitude et des différents mécanismes d'endommagement.

$9410 \mathrm{MPa}$ et de $9100 \mathrm{MPa}$, ce qui veut dire que la qualité d'interface a une influence sur le module d'élasticité. Le même constat a été fait par Di Liello et al. [5], dont les travaux portent sur l'effet du traitement des fibres de verre courtes et longues sur le comportement du polypropylène, et par Beaudoin [6], qui a étudié l'influence de la qualité de l'interface sur le comportement du PBT renforcé de billes ou de fibres de verre courtes. Ils en ont conclu tous deux que le traitement des fibres a pour conséquence de rigidifier le comportement élastique du matériau.

La qualité de l'interface fibre/matrice a une forte influence sur la contrainte maximale et la déformation à rupture. La contrainte maximale est 2 fois plus élevée pour le matériau avec fibres traitées alors que la déformation à rupture est 5 fois plus élevée pour le matériau avec fibres non-traitées. Ceci a été également constaté par Bounouas [1] pour les taux de fibres de $20 \%$ et $30 \%$, avec des différences plus élevées.

\subsubsection{Influence de la qualité de l'interface sur l'endommagement}

\subsubsection{1 Émission acoustique}

La mesure de l'émission acoustique permet d'identifier le niveau de contrainte auquel apparaît l'endommagement pour chacun des matériaux étudiés mais également, de quantifier chaque mécanisme d'endommagement intervenant dans la ruine des matériaux. Il est donc possible d'associer à chaque plage d'amplitude un mécanisme d'endommagement [7,8] (Fig. 5).
Les mécanismes d'endommagement apparaissent chronologiquement lors de la ruine du matériau. Il est à noter que le frottement avec glissement fibre/matrice n'est pas un endommagement car il n'induit pas de ruine du matériau. En effet, dans un premier temps il y a apparition de microfissures au sein de la matrice. Ces microfissures vont ensuite se regrouper (coalescence des microfissures) afin de créer des fissures, puis il y a rupture de l'interface fibre-matrice. L'interface étant rompue, il y a déchaussement des fibres suivi du frottement fibrematrice. Enfin, il y a rupture des fibres pour celles qui ne se sont pas déchaussées. L'association des différentes plages d'amplitudes aux différents mécanismes d'endommagement a été réalisée à partir de nombreux travaux traitant d'essais utilisant la technique d'émission acoustique couplée à des observations au M.E.B. [7]

On analyse les différents mécanismes intervenant lors de l'essai de traction jusqu'à rupture, pour une vitesse de $1,15 \times 10^{-04} \mathrm{~s}^{-1}$. Le tableau 3 , qui donne pour chaque plage d'amplitude et pour chacun des matériaux, le nombre d'événements acoustiques et le taux de participation, permettant d'apprécier la contribution de chaque mécanisme d'endommagement.

L'analyse des différentes plages d'amplitude donne :

33-45 dB : Cette plage d'amplitude caractérise la microfissuration de la matrice. Le matériau avec fibres traitées émet 2,9 fois plus d'événements acoustiques, à cause de la présence de l'interphase. Cela implique un niveau de contrainte d'apparition d'émission acoustique supérieur pour le matériau avec fibres traitées (30 MPa en moyenne), engendrant des contraintes de cisaillement locales plus élevées dans la matrice, et par conséquent un nombre de microfissures plus élevé (51041 événements) comparé au matériau avec fibres non-traitées (17543 événements).

En ce qui concerne le taux de participation, il est un peu plus élevé pour le matériau avec fibres non-traitées $(64,34 \%$ pour le matériau avec fibres traitées et $68,73 \%$ pour le matériau avec fibres non-traitées). 
On constate finalement que la microfissuration de la matrice est le mécanisme d'endommagement participant le plus à l'endommagement des matériaux.

45-58 dB : Cette plage d'amplitude caractérise la coalescence des microfissures. Il y a environ 3,6 fois plus d'événements pour le matériau avec fibres traitées. Un écart est également constaté entre les différents taux de participation. En effet, le taux de participation est plus élevé pour le matériau avec fibres traitées (33,1\%) qu'avec fibres non-traitées $(28,86 \%)$ donnant une différence de 4,24\%. La coalescence des microfissurations est le mécanisme d'endommagement apparaissant après la microfissuration de la matrice. Cela explique qu'il y a plus d'événements pour le matériau avec fibres traitées car dans la plage précédente (plage d'amplitude 33$45 \mathrm{~dB}$ ), il y avait plus d'événements pour ce matériau (51041 contre 17543 pour le matériau avec fibres non-traitées).

58-68 dB : Cette plage caractérise la rupture de l'interface. On constate qu'il y a 3,75 fois plus d'événements acoustiques pour le matériau avec fibres traitées. Par contre, le taux de participation de ce mécanisme d'endommagement est pratiquement le même pour les deux matériaux. De même, lors de la rupture de l'interface, on continue à enregistrer d'autres événements appartenant aux deux plages d'émission acoustique précédentes.

68-86 dB : Cette plage d'amplitude caractérise le frottement fibres/matrice et le déchaussement des fibres. Les deux phénomènes sont indissociables techniquement et seul le déchaussement des fibres est un mécanisme d'endommagement. Le frottement fibre/matrice et le déchaussement des fibres se fait uniquement sur les fibres dont l'axe d'orientation est confondu avec l'axe de traction. Les fibres étant réparties aléatoirement, il est normal d'obtenir une différence dans le nombre d'événements entre les deux matériaux.

87-100 dB : Cette plage d'amplitude caractérise la rupture des fibres de verre courtes. Lors de la rupture des matériaux, seules les fibres qui ne sont pas orientées suivant l'axe de traction cassent sous l'effet de la contrainte de cisaillement. Il y a peu d'événements pour les deux matériaux car lorsque le matériau rompt, les fibres soumises au cisaillement cassent pratiquement simultanément et par conséquent, la chaîne d'émission acoustique ne peut comptabiliser qu'une partie des événements acoustiques. Il y a donc un manque d'informations quantitatives pour cette plage d'amplitude.
L'émission acoustique ayant donné des informations quantitatives et qualitatives durant les essais, on utilise le microscope électronique à balayage afin de compléter les informations qualitatives en observant les faciès de rupture.

\subsection{Observation au microscope électronique à balayage (M.E.B.)}

Les observations au M.E.B. vont permettre de donner des informations supplémentaires sur l'influence qu'a l'ensimage des fibres sur les mécanismes d'endommagement.

* Matériau avec un taux de $40 \%$ en masse de fibres traitées (Figs. 6, 7).

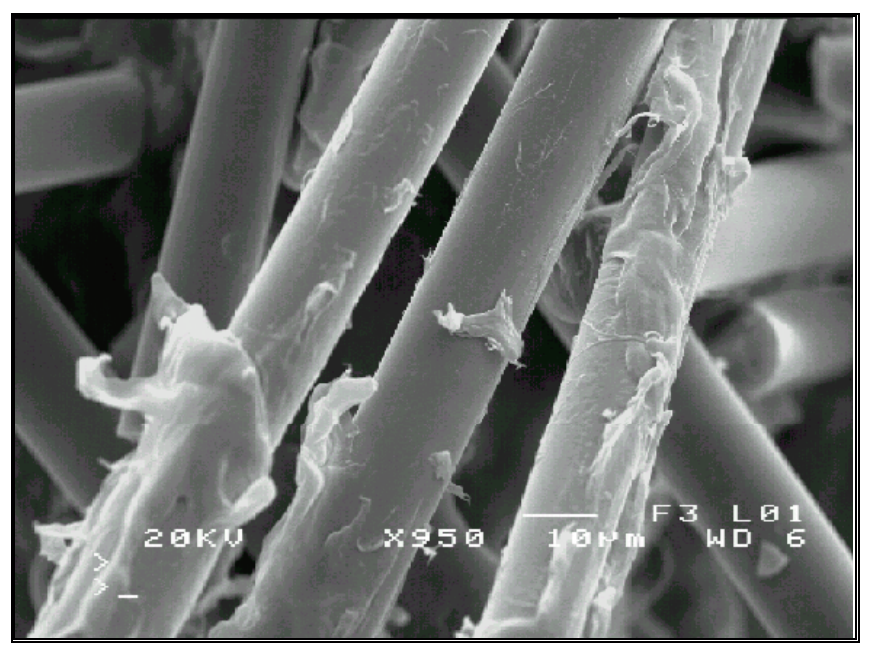

Fig. 6. Matériau avec fibres traitées.

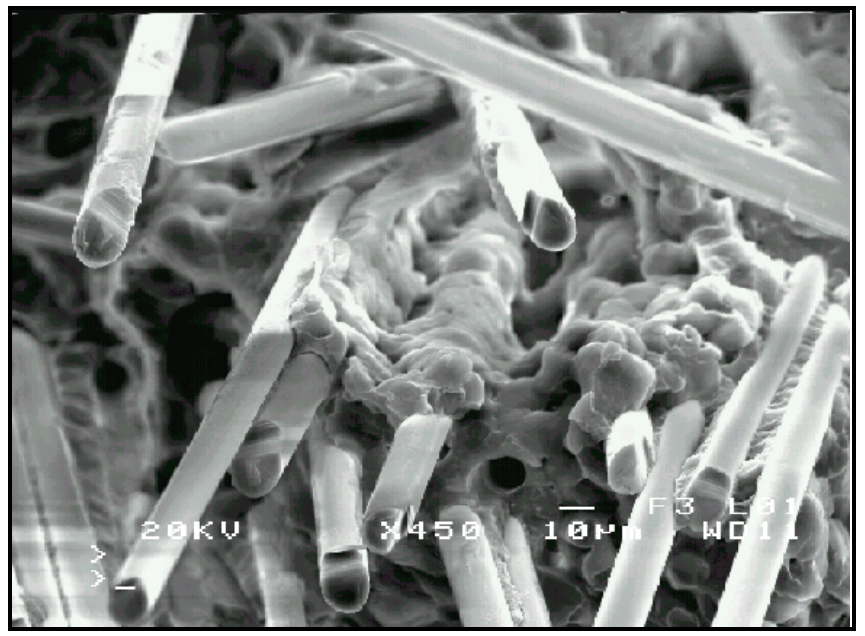

Fig. 7. Matériau avec fibres traitées.

On observe sur les figures 6 et 7 , la présence de polypropylène sur les fibres, qui est la conséquence du traitement chimique des fibres. Ce dernier permet un bon 
accrochage de la matrice sur les fibres, et rend le matériau plus résistant.

* Matériau avec un taux de $40 \%$ en masse fibres nontraitées (Figs. 8, 9).

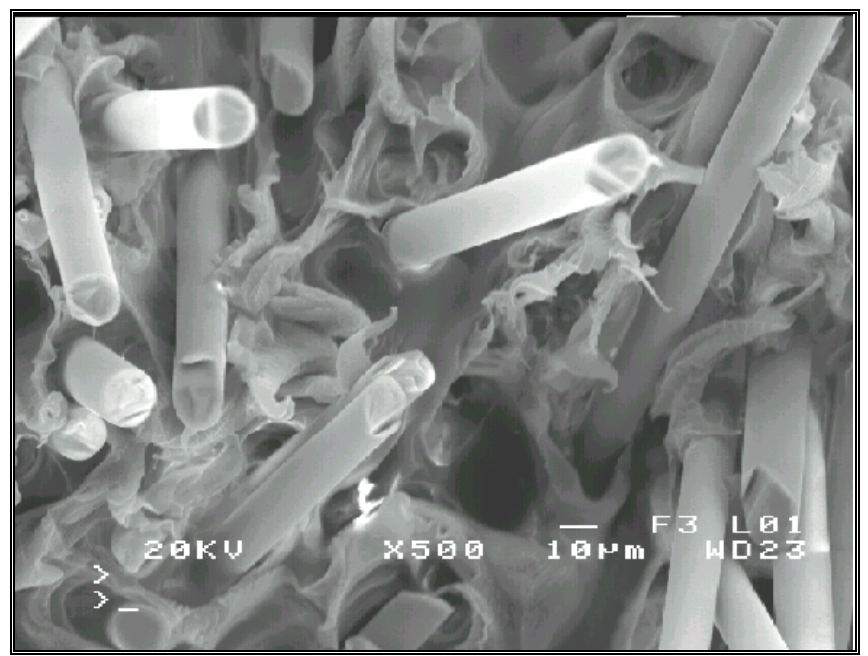

Fig. 8. Matériau avec fibres non-traitées.

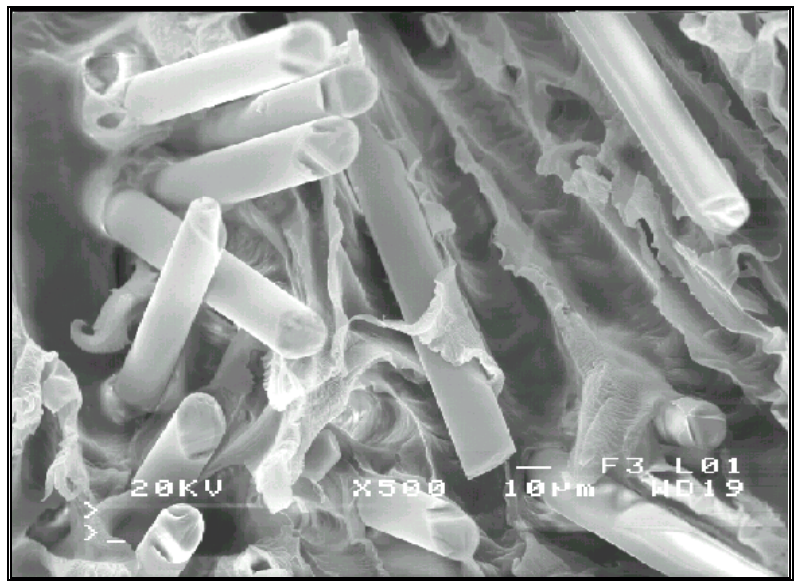

Fig. 9. Matériau avec fibres non-traitées.

On constate sur les figures 8 et 9, qu'il n'y a pas de polypropylène sur les fibres non-traitées et qu'elles ne présentent donc pas un bon accrochage avec la matrice.

\subsubsection{Conclusion sur les essais de traction}

On peut conclure, suite aux essais de traction monotones jusqu'à rupture réalisés sur le polypropylène chargé de fibres de verre courtes traitées et non-traitées pour un taux de masse de $20 \%, 30 \%$ et $40 \%$, que le meilleur taux de fibre est de $40 \%$. Les propriétés du matériau, notamment pour celui avec fibres non-traitées, évolueront faiblement si on augmente le taux de fibres au-delà de $40 \%$.
De même, la qualité de l'interface fibre-matrice a une influence sur le comportement mécanique ainsi que sur les mécanismes d'endommagement. Ségard [2,3] a montré que le niveau de déformation visqueux pour le matériau avec fibres traitées est moins important. Pour cela, les modèles de Schapery [9] et de Zapas-Crissman [10] ont été utilisés dans la simulation de fluage-recouvrance. Cela veut dire que la matrice du matériau avec fibres nontraitées a un comportement visqueux plus prononcé ce qui a une influence sur le nombre d'événements acoustiques. En effet, on constate, pour chaque plage d'amplitude, que le nombre d'événements acoustiques est plus élevé pour le matériau avec fibres traitées. De même, pour les deux types d'interfaces, la majorité des événements acoustiques appartiennent à la plage $35-45 \mathrm{~dB}$ caractérisant la microfissuration de la matrice suivie de la plage 45-58 dB caractérisant la coalescence des microfissurations.

\subsection{Essais de fluage jusqu'à rupture}

\subsubsection{Conditions d'essais}

Les essais de fluage sont réalisés sur une machine de type MAYES TC 30. Cette machine de fluage est équipée d'un four régulé, et est prévue à l'origine pour faire des essais de fluage sur des éprouvettes métalliques de forme cylindrique. Le niveau de charge est fixé par des masses suspendues à un levier. Pour libérer la charge, il suffit d'amener le support de poids jusqu'aux masses, afin de les soutenir et par conséquent d'annuler l'effort engendré par celles-ci sur l'éprouvette testée (voir Fig. 10).

Tout comme les essais de traction monotone, les essais sont réalisés à température ambiante $\left(19^{\circ} \mathrm{C}\right)$ sur le polypropylène renforcé de $40 \%$ en masse de fibres de verre courtes traitées et non-traitées. Le suivi de l'endommagement se fait à l'aide de la mesure de l'émission acoustique. Le niveau de charge choisi est situé à $90 \%$ de la charge à rupture en traction (ce qui donne pour le matériau avec fibres non-traitées, une contrainte de $31 \mathrm{MPa}$, et pour le matériau avec fibres traitées, une contrainte de $66 \mathrm{MP}$ ) afin que les essais soient de courte durée pour ne pas avoir de problème de mémoire lors de l'acquisition des données (échantillonnage de $100 \mathrm{~ms}$ ).

\subsubsection{Résultats et discussion}

Il est impossible de faire une comparaison des résultats par rapport au niveau des déformations sur le matériau avec fibres traitées et non-traitées, car les essais de fluage jusqu'à rupture sur les deux matériaux se font à des niveaux de contraintes appliquées totalement différents. L'analyse se fera donc uniquement sur l'identification et la quantification des mécanismes d'endommagement. Par contre, en appliquant une charge à $90 \%$ de la charge à rupture pour les deux matériaux, le temps à rupture est quasiment identique et est de l'ordre de $15 \mathrm{~min}$ en moyenne. 
Tableau 4. Nombre d'événements et taux de participation par plage d'amplitude pour les matériaux avec fibres traitées et non-traitées pour un taux de fibres en masse de $40 \%$ soumis à un essai de fluage jusqu'à rupture.

\begin{tabular}{|c|c|c|c|c|}
\hline & \multicolumn{2}{|c|}{$\begin{array}{l}\text { Matériau avec fibres traitées } \\
\qquad \sigma=69 \mathrm{MPa}\end{array}$} & \multicolumn{2}{|c|}{$\begin{array}{l}\text { Matériau avec fibres non-traitées } \\
\qquad \sigma=28 \mathrm{MPa} \\
\end{array}$} \\
\hline & $\begin{array}{c}\text { Nombre } \\
\text { d'événements }\end{array}$ & $\begin{array}{c}\text { Taux } \\
\text { de participation }\end{array}$ & $\begin{array}{c}\text { Nombre } \\
\text { d'événements }\end{array}$ & $\begin{array}{c}\text { Taux } \\
\text { de participation }\end{array}$ \\
\hline $33-45 \mathrm{~dB}$ & 125198 & $79,33 \%$ & 51104 & $76,31 \%$ \\
\hline $45-58 \mathrm{~dB}$ & 29858 & $18,92 \%$ & 11580 & $17,29 \%$ \\
\hline $58-68 \mathrm{~dB}$ & 1835 & $1,16 \%$ & 719 & $1,07 \%$ \\
\hline $68-86 \mathrm{~dB}$ & 630 & $0,4 \%$ & 882 & $1,32 \%$ \\
\hline 86-100 dB & 291 & $0,19 \%$ & 2686 & $4,01 \%$ \\
\hline Total & 157812 & $100 \%$ & 66971 & $100 \%$ \\
\hline
\end{tabular}

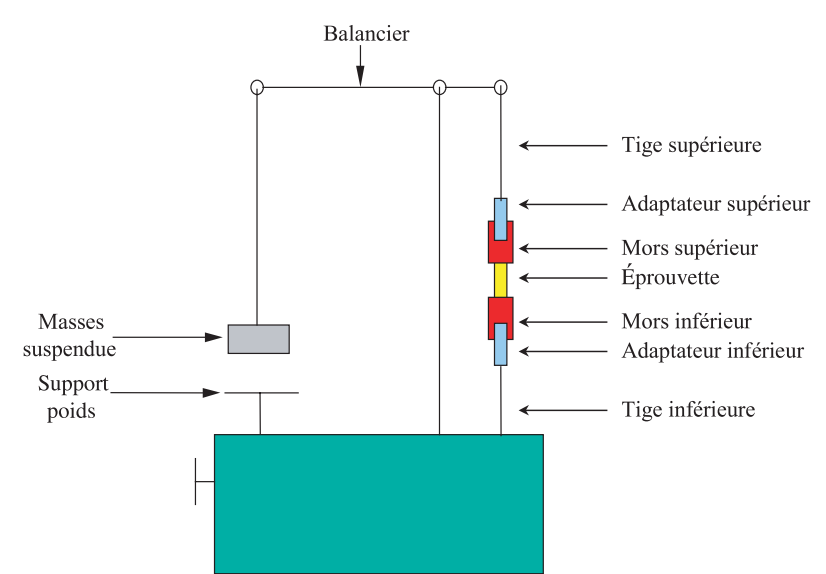

Fig. 10. Schéma de la machine de fluage modifiée.

Tout comme pour les essais de traction monotone, on analyse les différents mécanismes intervenant lors des essais de fluage jusqu'à rupture pour chacun des matériaux.

L'analyse des différentes plages d'amplitude donne :

33-45 dB : Cette plage d'amplitude caractérise la microfissuration de la matrice. Le matériau avec fibres traitées émet 2,6 fois plus d'événements acoustiques, du fait de la présence de l'interphase. Le nombre de microfissures est plus élevées pour celui-ci (125 198 événements) comparé au matériau avec fibres non-traitées (51 104 événements). On constate que la microfissuration de la matrice est le mécanisme d'endommagement participant le plus à l'endommagement des matériaux (79,33\% pour le matériau avec fibres traitées et 76,31\% pour le matériau avec fibres non-traitées).

45-58 dB : Cette plage d'amplitude caractérise la coalescence des microfissures. La coalescence des microfissures est le mécanisme d'endommagement intervenant juste après la microfissuration de la matrice. Il y a 2,6 fois plus d'événements pour le matériau avec fibres traitées. Ceci est la conséquence d'un nombre plus élevé d'événements caractérisant la microfisuration de la matrice.

58-68 dB : Cette plage caractérise la rupture de l'interface. On constate qu'il y a 2,6 fois plus d'événements acoustiques pour le matériau avec fibres traitées. Par contre, tout comme les essais de traction, le taux de participation de ce mécanisme d'endommagement est pratiquement le même pour les deux matériaux.

68-86 dB : Cette plage d'amplitude caractérise le frottement fibres/matrice et le déchaussement des fibres. Comme il a été spécifié pour les essais de traction, les deux phénomènes sont indissociables techniquement et seul le déchaussement des fibres est un mécanisme d'endommagement. Le frottement fibre/matrice et le déchaussement des fibres se fait uniquement sur les fibres dont l'axe d'orientation est confondu avec l'axe de traction en fluage. Les fibres étant réparties aléatoirement, il est normal d'obtenir une différence dans le nombre d'événements entre les deux matériaux.

87-100 dB : Cette plage d'amplitude caractérise la rupture des fibres de verre courtes. Tout comme les essais de traction monotone, lors de la rupture des matériaux, seules les fibres qui ne sont pas orientées suivant l'axe de traction lors du fluage cassent.

\subsection{Influence de la nature de la sollicitation sur les mécanismes d'endommagement}

Si on compare les résultats de l'émission acoustique obtenus à partir des essais de traction monotone du tableau 3, aux résultats obtenus à partir des essais de fluage en traction du tableau 4, on constate, au vu du nombre d'événements total, qu'il y a plus d'événements pour le matériau soumis au fluage en traction ( 2 fois plus pour le matériau avec fibres traitées et 2,5 fois plus pour le matériau avec fibres non-traitées). La plus grande différence est constatée pour la plage $33-45 \mathrm{~dB}$, car la 
majorité des événements appartient à cette plage et caractérisent la microfissuration de la matrice. De même, le taux de participation dans cette plage est plus élevée pour les essais de fluage (écart de 12,45\% en plus en moyenne).

Ainsi, le type de sollicitation a une influence sur la cinétique de l'endommagement de chaque matériau. Ceci est dû à la différence de vitesse de déformation qui est constante pour les essais de traction monotone, et qui par contre, ne l'est pas lors des essais de fluage en traction.

\section{Conclusion}

L'investigation du polypropylène renforcé de fibres de verre courtes a permis de mettre en évidence, grâce aux essais de traction monotone, l'influence du taux de fibres sur le comportement mécanique du matériau. Le taux de fibre seuil, au-delà duquel il n'y a quasiment plus d'évolution des propriétés mécaniques, est de $40 \%$. De même, pour les deux types d'interface, il y a apparition de 3 zones de comportement distinctes qui sont linéaire, non-linéaire sans endommagement et non-linéaire avec endommagement, le matériau ayant les meilleures propriétés mécaniques étant celui avec les fibres traitées. La mesure de l'émission acoustique a permis de mettre en évidence dans un premier temps l'importance de la qualité de l'interface sur les mécanismes d'endommagement. Il a été constaté qu'il y a plus d'événements acoustiques pour le matériau avec fibres traitées. Dans un deuxième temps, on observe pour les deux matériaux, que les mécanismes d'endommagements prépondérants sont la microfissuration de la matrice et la coalescence de ces microfissures.

Les essais de fluage jusqu'à rupture ont montré, tout comme pour les essais de traction monotone, qu'il y a plus d'événements acoustiques pour le matériau avec fibres traitées et une prépondérance, pour les deux types de matériau, des mécanismes d'endommagement caractérisant la microfissuration de la matrice ainsi que la coalescence des microfissures.

La comparaison des résultats montre, pour les deux types de matériau, qu'il y a plus d'événements acoustiques pour la sollicitation en fluage.

\section{Références}

[1] L. Bounouas, Étude phénoménologique de l'influence de paramètres structuraux et de mise en œuvre sur le comportement mécanique du polypropylène chargé de fibres courtes, Thèse de Doctorat, Université de Technologie de Compiègne, 2001

[2] E. Ségard, Étude expérimentale et théorique de l'influence de la qualité de l'interface fibre/matrice sur le comportement en fluage d'un polypropylène renforcé de fibres de verre courtes, Thèse de Doctorat, Université de Technologie de Compiègne, Novembre 2001

[3] E. Ségard, S. Benmedakhene, A. Laksimi, Influence of the fibre-matrix interface on the behaviour of polypropylene reinforced by short glass fibres above glass transition temperature, Composites Science and Technology 62(15) (2002) 2029-2036

[4] E. Ségard, S. Benmedakhene, A. Laksimi, D. Laï, Influence of interface on the behaviour of polypropylene reinforced with short glass fibers, 13th International Conference on Composite Materials, Pékin, China, 25-29 June 2001

[5] V. Di Liello, E. Martuscelli, G. Ragosta, A. Zihlif, Effect of fibre surface treatment on yielding and fracture behaviour of glass fibre - polypropylene composite, J. Mat. Sci. 26 (1991) 2100-2106

[6] O. Beaudoin, Contribution à l'étude des relations microstructure - Propriétés mécaniques d'une matrice polybutylène terephtalate $(\mathrm{PBT})$ renforcé de billes ou de fibres de verre, Thèse de Doctorat, Université de Montpellier II, Mars 1998

[7] S. Barré, M. Benzeggagh, On the use of acoustic emission to investigate damage mechanics in glass-fibre reinforced polypropylene, Composites Science and Technology 52 (1994) 369-376

[8] X.L. Gong, A. Laksimi, M. Benzeggagh, New approach of the acoustic emission and its application to the identification of the damage mechanisms in composite materials, Revue des Composites et des Matériaux Avancés 8-1 (1994) 7-23

[9] R.A. Schapery, On the caracterization of nonlinear viscoelastic materials, Polymer Engineering and Science 9 (1969) 295-310

[10] L.J. Zapas, J.M. Crissman, Creep and recovery behaviour of ultra-high molecular weight polythylene in the region of small uniaxial deformations, Polymer 25 (1984) 57-62 